\title{
The Wild Yeasts Biodiversity Project
}

\section{Sarah R. Stockwell}

\author{
University of California, San Diego; Section of Ecology, Behavior and Evolution, Division of \\ Biology, 9500 Gilman Dr, La Jolla CA 92093-0116 USA \\ (sarahs@ucsd.edu)
}

\section{Extended Abstract}

The Wild Yeast Biodiversity Project is an inquiry-based module developed for Molecular Methods in Evolution and Ecology, an upper-division biology lab class at the University of California, San Diego. It serves as one of three major projects that student complete over the course of the 10-week class. In the project, students work to isolate strains of wild yeast from the chaparral at a local nature reserve. They take field samples, culture the samples to isolate individual strains of yeast, and identify the strains using DNA barcoding. The project has 4 goals:

1) Guide students through an authentic process of scientific discovery in which they collect novel data and contribute to our understanding of microbial diversity and ecology.

2) Teach students scientific skills: field collecting, microbiology culturing, molecular biology techniques, basic bioinformatics skills, microbiology safety, and aseptic technique.

3) Build a living archive of the fungal diversity at the Scripps Coastal Reserve.

4) Discover and document new species of wild yeasts, in collaboration with the Hittinger Lab at the University of Wisconsin-Madison.

On the initial collecting trip, students are introduced to the coastal sage scrub habitat, an important and threatened ecosystem. They learn to identify the most common native plants in our region and find out about the plants' adaptations to San Diego's Mediterranean-type ecosystem. Then they set off in pairs to explore the habitat and choose their own samples. Most students sample plant tissue - leaves, fresh and dried flowers, decaying prickly pear pads, etc. - but some sample the arthropods they find, such as bees or caterpillars. Each individual student aseptically collects seven samples. By this point, some students are already articulating scientific questions: Will the fresh and dried flowers of the same plant yield different yeasts? Will leaves yield fewer yeasts than flowers do?

Back at the lab, students incubate their samples in liquid media that they make and sterilize themselves. The culturing conditions favor the growth of fungi and discourage bacteria: limited oxygen (capped tubes, no agitation), high osmotic stress ( $8 \%$ glucose), and antibiotics. The cultures go through two rounds of liquid media and then multiple rounds of agar plate culture, as students try to isolate individual yeast strains from the diverse community of microbes that are originally present on each sample. During this process, students learn microbiology culturing techniques, including proper streaking technique, and gain experience in identifying yeast morphology. Each student starts with 7 samples and ends up with an average of 2-3 isolated strains.

Throughout the culturing phase of the project, we strongly emphasize microbiology safety and implement Biosafety Level 2 protocols. Yeast are rarely pathogenic compared to bacteria and mold, but we take extra precautions because the strains are unidentified at this point. Former students have reported that these skills proved useful in their subsequent positions in research labs.

All yeast strains are preserved for future study, building a living archive of microbial biodiversity at the nature reserve that also serves to document changes over time in this ecologically sensitive habitat. The students prepare a sample of each isolated culture for cryogenic storage at $-80^{\circ} \mathrm{C}$. After three iterations of the course, in early spring of 2017, 2018, and 2019, we have accumulated 377 frozen isolates from the natural reserve area. This longitudinal data set will be a rich resource for testing ecological and evolutionary questions.

Each student extracts genomic DNA from three of their isolated cultures, amplifies the ITS/5.8S ribosomal barcoding region via PCR, and runs the results on a gel. The procedure is unusually robust; most students get clear, isolated bands for at least two of their strains, and often for all three. Students choose their two favorite successful 
strains and we send them out for Sanger sequencing. Students analyze the resulting sequences and try to identify their species using online resources. Each student writes up a "yeast portfolio" detailing all the information they are able to find about their species.

Once students have identified their species, the class pools its data. Students work in pairs to look for patterns in the pooled data, such as associations between plant species and yeast taxa. They then write and present a short proposal for further research to test the pattern they found.

This lab module has been developed in collaboration with Chris Hittinger at the University of WisconsinMadison, whose lab developed the original protocols (Sylvester et al. 2015) and whose advice has been invaluable. I have expanded the protocols into a 50-page lab manual that guides students through collecting, culturing, phenotyping, isolating, freezing, DNA barcoding, and identifying their wild yeast strains. The UCSD course also includes additional elements to situate the experiments within the process of science, such as the research proposal.

Microbial ecology is understudied, and scientists have identified a small fraction of the world's yeast species. Over three iterations of the lab class, students have found twelve yeast strains that have good-quality sequences but do not have species-level matches in GenBank, making them potentially novel species. We are sharing this data with the Hittinger lab, which has the capacity to fully phenotype and formally describe new yeast species. In addition, we are sharing our data on identified yeasts so they can be added to the database of wild yeasts maintained by the Hittinger Lab, where they can be used by other researchers.

Keywords: microbiology, evolution, ecology, natural history, CURE, inquiry-based learning

Link to Original Poster File: https://doi.org/10.37590/able.v41.poster83

\section{Cited References}

Sylvester K, Wang QM, James B, Mendez R, Hulfachor AB, Hittinger CT. 2015. Temperature and host preferences drive the diversification of Saccharomyces and other yeasts: a survey and the discovery of eight new yeast species. FEMS Yeast Research. 5(3):fov002.

\section{Mission, Review Process \& Disclaimer}

The Association for Biology Laboratory Education (ABLE) was founded in 1979 to promote information exchange among university and college educators actively concerned with teaching biology in a laboratory setting. The focus of ABLE is to improve the undergraduate biology laboratory experience by promoting the development and dissemination of interesting, innovative, and reliable laboratory exercises. For more information about ABLE, please visit http://www.ableweb.org/.

Advances in Biology Laboratory Education is the peer-reviewed publication of the conference of the Association for Biology Laboratory Education. Published articles and extended abstracts are evaluated and selected by a committee prior to presentation at the conference, peer-reviewed by participants at the conference, and edited by members of the ABLE Editorial Board. Published abstracts are evaluated and selected by a committee prior to presentation at the conference.

\section{Citing This Article}

Stockwell SR. 2020. The wild yeasts biodiversity project. Article 83 In: McMahon K, editor. Advances in biology laboratory education. Volume 41. Publication of the 41st Conference of the Association for Biology Laboratory Education (ABLE). https://doi.org/10.37590/able.v41.extabs83

Compilation (C) 2020 by the Association for Biology Laboratory Education, ISBN 1-890444-17-0. All rights reserved. No part of this publication may be reproduced, stored in a retrieval system, or transmitted, in any form or by any means, electronic, mechanical, photocopying, recording, or otherwise, without the prior written permission of the copyright owner.

ABLE strongly encourages individuals to use the exercises in this volume in their teaching program. If this exercise is used solely at one's own institution with no intent for profit, it is excluded from the preceding copyright restriction, unless otherwise noted on the copyright notice of the individual chapter in this volume. Proper credit to this publication must be included in your laboratory outline for each use; a sample citation is given above. 
Stockwell

Publication of Association for Biology Laboratory Education, Volume 41, 2020 